\title{
Encorafenib/binimetinib for the treatment of BRAF-mutant advanced, unresectable, or metastatic melanoma: design, development, and potential place in therapy
}

REVIEW

This article was published in the following Dove Press journal:

OncoTargets and Therapy

James Sun

Jonathan S Zager

Zeynep Eroglu

Department of Cutaneous Oncology,

$\mathrm{H}$. Lee Moffitt Cancer Center and Research Institute, Tampa, FL, USA
Correspondence: Zeynep Eroglu Department of Cutaneous Oncology, $\mathrm{H}$. Lee Moffitt Cancer Center and Research Institute, 10920 McKinley Drive, Tampa, FL 336I2, USA

Tel + I 8137457488

Fax +I 8137455725

Email zeynep.eroglu@moffitt.org

\begin{abstract}
Major advances in the understanding of the pathophysiology of melanoma have led to a new era of melanoma treatment with targeted therapy and immunotherapies. Since 2011, four new classes of medications with unique mechanisms of action have been approved, which allow melanoma to be treated at many different stages in its development. These include the checkpoint inhibitors anti-PD1/PDL-1 and anti-CTLA4, as well as BRAF inhibitors and MEK inhibitors. The latter two were developed to directly inhibit key components in the MAP kinase pathway with significant breakthrough in the treatment of metastatic and unresectable melanoma. In this review, we discuss the development of targeted therapy of melanoma up to the latest agents encorafenib and binimetinib, including mechanisms of action, adverse effects, and the latest data on treatment response. Current ongoing trials will continue to elucidate these medications and their ultimate impact on melanoma therapy.
\end{abstract}

Keywords: encorafenib, binimetinib, LGX818, MEK162, advanced melanoma, BRAF, MEK

\section{Introduction}

Malignant melanoma therapy has undergone a revolution in the last several years. It is now well established that $\sim 40 \%-50 \%$ of all melanomas have a serine-threonine protein kinase B-RAF (BRAF) mutation. ${ }^{1-3}$ The most commonly observed BRAF mutations are due to single nucleotide substitutions of glutamic acid. Over $90 \%$ are due to glutamic acid for valine (BRAFV600E). ${ }^{2}$ The second most common mutation is BRAFV $600 \mathrm{~K}$, lysine for valine, representing $\sim 5 \%-6 \%$ of the mutations. Other rarely observed mutations include BRAFV600D, V600R, or even two-nucleotide variations. ${ }^{2,4}$ RAS mutations were also identified in melanoma cells. NRAS mutations are found in approximately $15 \%$ of all melanomas at diagnosis and in most cases are mutually exclusive with BRAF mutations. ${ }^{1,5,6}$ Both NRAS and BRAF mutations cause unchecked activation of the MAP kinase signal transduction pathway (RAS-RAF-MEK-ERK), leading to unregulated growth of tumor cells. ${ }^{1,5,7}$ Understanding of this pathway has led to the identification of specific targets for therapy.

\section{Development of BRAF inhibitors}

With the discovery of BRAF mutations in $2002,{ }^{1}$ the first approved second-generation mutant BRAF-specific inhibitors were created using scaffold-based crystallography. ${ }^{8,9}$ This compound, vemurafenib (Zelboraf ${ }^{\circledR}$, PLX4032; Plexxikon, Berkeley, CA, USA), 
was approved in 2011 for the treatment of BRAFV600E metastatic melanoma. The Phase III BRIM3 trial compared vemurafenib and dacarbazine (Table 1). ${ }^{10}$ The objective response rate for vemurafenib was 48\% (95\% CI: 42-55) compared to $5 \%$ (95\% CI: 3-9) for dacarbazine $(P<0.001)$. Median progression-free survival (PFS) was 5.3 months vs 1.6 months (HR 0.26, 95\% CI: $0.20-0.33$ ). The RR reduction for death or disease progression was $74 \%$ for vemurafenib compared to dacarbazine. ${ }^{10,11}$ The next second-generation BRAF inhibitor was dabrafenib (Tafinlar ${ }^{\circledR}$, GSK2118436; GlaxoSmithKline plc, London, UK), approved in 2013 for the treatment of both V600E/K-mutated melanomas. ${ }^{3}$ Dabrafenib was similarly compared to dacarbazine in a Phase III trial that again confirmed the superiority of BRAF inhibitor treatment

Table I Comparison of clinical trials studying BRAF/MEK inhibitors

\begin{tabular}{|c|c|c|c|c|}
\hline Trial & Drug & $\begin{array}{l}\text { Median PFS } \\
\text { (months) }\end{array}$ & $\begin{array}{l}\text { Median OS } \\
\text { (months) }\end{array}$ & Adverse effects \\
\hline BRIM3 $^{10}$ & Vemurafenib & 5.3 & 13.6 & $\begin{array}{l}\text { Most common: cutaneous events (photosensitivity, blistering), } \\
\text { arthralgia, and fatigue } \\
\text { Major: arthralgia, rash, fatigue, cutaneous squamous-cell } \\
\text { carcinoma, keratoacanthoma, nausea, pruritis, hyperkeratosis, } \\
\text { diarrhea, headache, vomiting, and neutropenia }\end{array}$ \\
\hline BREAK-3'2 & Dabrafenib & 5.1 & 20 & $\begin{array}{l}\text { Most common: cutaneous events (hyperkeratosis, papillomas, } \\
\text { PPED), pyrexia, fatigue, headache, and arthralgia } \\
\text { Major: hyperkeratosis, PPED, cSCC, keratoacanthoma, nausea, } \\
\text { vomiting, neutropenia, and thrombocytopenia }\end{array}$ \\
\hline METRIC $^{44}$ & Trametinib & 4.8 & N/A & $\begin{array}{l}\text { Most common: rash (papulopustular), diarrhea, peripheral } \\
\text { edema, fatigue, and dermatitis acneiform } \\
\text { Major: rash, fatigue, peripheral edema, acneiform dermatitis, } \\
\text { nausea, alopecia, hypertension, constipation, central serous } \\
\text { retinopathy, and retinal vein occlusion }\end{array}$ \\
\hline \multirow[t]{2}{*}{ COMBI-d ${ }^{35}$} & Dabrafenib & 8.8 & 18.7 & $\begin{array}{l}\text { Most common: hyperkeratosis, fatigue, PPED, alopecia, pyrexia, } \\
\text { arthralgia } \\
\text { Major: pyrexia, chills, fatigue, rash, nausea, diarrhea, vomiting, } \\
\text { hyperkeratosis, and PPED }\end{array}$ \\
\hline & $\begin{array}{l}\text { Dabrafenib + } \\
\text { trametinib }\end{array}$ & 11 & 25.1 & $\begin{array}{l}\text { Most common: pyrexia, chills, fatigue, rash, and nausea } \\
\text { Fewer cSCC, hyperkeratosis, skin papillomas, alopecia, } \\
\text { and PPED } \\
\text { Pyrexia more common } \\
\text { Major: pyrexia, fatigue, diarrhea, arthralgia, vomiting, peripheral } \\
\text { edema, and PPED }\end{array}$ \\
\hline \multirow[t]{2}{*}{ COMBI-v² } & Vemurafenib & 7.3 & 18 & $\begin{array}{l}\text { Most common: arthralgia, rash, alopecia, diarrhea, nausea, } \\
\text { and fatigue } \\
\text { Major: pyrexia, nausea, diarrhea, vomiting, arthralgia, } \\
\text { rash, alopecia, PPED, hyperkeratosis, skin papilloma, } \\
\text { and photosensitivity }\end{array}$ \\
\hline & $\begin{array}{l}\text { Dabrafenib + } \\
\text { trametinib }\end{array}$ & 11.4 & 25.6 & $\begin{array}{l}\text { Most common: pyrexia, nausea, diarrhea, chills, fatigue, } \\
\text { headache, and vomiting } \\
\text { Pyrexia more common } \\
\text { Fewer rash, photosensitivity, PPED, skin papillomas, cSCC, } \\
\text { keratoacanthoma, and hyperkeratosis } \\
\text { Major: pyrexia, nausea, diarrhea, chills, vomiting, arthralgia, } \\
\text { and rash }\end{array}$ \\
\hline $\operatorname{coBRIM}^{31}$ & Vemurafenib & 7.2 & 17.4 & $\begin{array}{l}\text { Most common: rash, arthralgia, diarrhea, fatigue, alopecia, } \\
\text { hyperkeratosis, nausea, pyrexia, decreased appetite, } \\
\text { photosensitivity, and serous retinopathy } \\
\text { Major: rash, arthralgia, diarrhea, fatigue, } \\
\text { alopecia, hyperkeratosis, nausea, decreased appetite, and } \\
\text { vomiting }\end{array}$ \\
\hline
\end{tabular}


Table I (Continued)

\begin{tabular}{|c|c|c|c|c|}
\hline Trial & Drug & $\begin{array}{l}\text { Median PFS } \\
\text { (months) }\end{array}$ & $\begin{array}{l}\text { Median OS } \\
\text { (months) }\end{array}$ & Adverse effects \\
\hline & $\begin{array}{l}\text { Vemurafenib + } \\
\text { cobimetinib }\end{array}$ & 12.3 & 22.3 & $\begin{array}{l}\text { Most common: rash, diarrhea, nausea, arthralgia, fatigue, } \\
\text { photosensitivity, pyrexia, vomiting, serous retinopathy, alopecia, } \\
\text { and hyperkeratosis } \\
\text { Fewer cSCC, keratoacanthoma, and Bowen's disease } \\
\text { Photosensitivity more common } \\
\text { Serous retinopathy, decreased LVEF, and increased CPK level }\end{array}$ \\
\hline \multirow{3}{*}{$\begin{array}{l}\text { COLUMBUS } \\
\text { part I }{ }^{34,36,37}\end{array}$} & Vemurafenib & 7.3 & 16.9 & Major: arthralgia \\
\hline & Encorafenib & 9.6 & N/A & Major: PPED, myalgia, arthralgia, vomiting, nausea \\
\hline & $\begin{array}{l}\text { Encorafenib + } \\
\text { binimetinib }\end{array}$ & 14.9 & 33.6 & $\begin{array}{l}\text { More common: GI (diarrhea, constipation, vomiting, abdominal } \\
\text { pain), asymptomatic CPK increase, and blurred vision } \\
\text { Less common: skin toxicity (pruritis, hyperkeratosis, rash, } \\
\text { keratosis pilaris, palmoplantar keratoderma, PPED, dry skin, } \\
\text { skin papilloma, maculopapular rash, and sunburn), alopecia, } \\
\text { photosensitivity, arthralgia, myalgia, extremity pain, decreased } \\
\text { appetite, musculoskeletal pain, and decreased weight } \\
\text { Major: elevated GGT, elevated CPK, HTN, and pyrexia }\end{array}$ \\
\hline \multirow[t]{2}{*}{$\begin{array}{l}\text { COLUMBUS } \\
\text { part } 2^{38}\end{array}$} & $\begin{array}{l}\text { Encorafenib } \\
(\text { Encorafenib } \\
\text { part I+2) }\end{array}$ & $\begin{array}{l}7.4 \\
9.2\end{array}$ & N/A & \\
\hline & $\begin{array}{l}\text { Encorafenib + } \\
\text { binimetinib }\end{array}$ & 12.9 & N/A & \\
\hline
\end{tabular}

Abbreviations: cSCC, cutaneous squamous cell carcinoma; GI, gastrointestinal; OS, overall survival; PPED, palmar-plantar erythrodysesthesia; LVEF, left ventricular ejection fraction; CPK, creatine phosphokinase; N/A, not applicable.

compared to chemotherapy. ${ }^{12}$ Median PFS was 5.1 months for dabrafenib vs 2.7 months for dacarbazine (HR 0.30, 95\% CI: $0.18-0.51 ; P<0.0001)$, and overall response rate (ORR) was $50 \%$ vs $3 \% .^{3,12}$

Despite the revolution in melanoma treatment with the discovery of BRAF inhibitors, the early aggressive response to therapy is tempered by rapid development of treatment resistance within 6-8 months. ${ }^{13-15}$ Several mechanisms of treatment resistance have been identified, but the most common mechanism is via reactivation of the MAPK pathway, termed paradoxical MAPK-pathway activation. . $^{3,11,13}$ While BRAF remains inhibited, other RAF proteins CRAF and ARAF can then replace BRAF and continue MAPK pathway hyperactivation., ${ }^{3,13}$ Constitutive activation of ERK ultimately leads to unregulated cell growth. ${ }^{14}$ Another consequence of paradoxical reactivation is the development of cutaneous squamous cell carcinoma (cSCC) and keratoacanthomas as an adverse reaction BRAF inhibitor therapy. ${ }^{3,15}$ Approximately $14 \%-26 \%$ of the patients treated with vemurafenib or dabrafenib developed these secondary skin cancers. ${ }^{3}$ While a full discussion of BRAF inhibitor resistance is outside the scope of this review, the mechanism of resistance is an important characteristic of the newest BRAF inhibitor to be discussed next.

\section{Encorafenib}

Encorafenib (Braftovi ${ }^{\mathrm{TM}}$, LGX818; Array BioPharma, Boulder, CO, USA) is a selective, ATP-competitive BRAF inhibitor for BRAFV600-mutated cells. ${ }^{2,15,17}$ It is the newest second generation of BRAF inhibitors and was recently FDA approved in advanced melanoma. Encorafenib is administered orally with $85 \%$ bioavailability, reaching maximum post-dose concentration in 2 hours with a 6-hour half-life. Food intake delays absorption of the medication but does not alter overall absorption. It is metabolized by cytochrome 450 enzymes (CYP3A4, CYP2C19, and CYP2D6) and 20 unique metabolites have been identified, which are excreted equally in urine and feces. Two percentage and 5\% of the absorbed medication is excreted unchanged in urine and feces, respectively. ${ }^{18}$

The most significant difference between encorafenib and the other second-generation BRAF inhibitors is its increased dissociation half-life of 30 hours, compared to dabrafenib ( 2 hours) and vemurafenib (0.5 hours).$^{15,18}$ This means that encorafenib will maintain its inhibitory effect longer than the other BRAF inhibitors with a shorter off-rate, leading to decreased off-effect adverse events (AEs) and prolonged inhibition. ${ }^{3}$ To confirm the long dissociation time, the inhibitory effect of encorafenib was indirectly investigated by 
measuring effective concentration (EC50) of phosphorylated ERK (pERK). These experiments showed a twofold change in pERK EC50 following washout, as compared to a 14 and 23 -fold shift for dabrafenib and vemurafenib, respectively. ${ }^{15}$ Encorafenib also demonstrates higher potency with a halfmaximal inhibitory concentration (IC50) of $<40 \mathrm{nmol} / \mathrm{L}$, compared to the higher IC50s of dabrafenib $(<100 \mathrm{nmol} / \mathrm{L})$ and vemurafenib $(<1 \mu \mathrm{mol} / \mathrm{L})$. The prolonged dissociation half-life and potency may translate into prolonged therapeutic effect with less toxicity. In addition to higher potency, encorafenib is also a highly specific BRAF inhibitor, only demonstrating inhibition against one kinase other than mutated and wild-type BRAF out of a panel of 99 different kinases. ${ }^{15,18}$

Delord et a ${ }^{15}$ performed a randomized Phase I doseescalation and expansion trial of encorafenib that demonstrated effective tumor growth inhibition in murine models at doses as low as $5 \mathrm{mg} / \mathrm{kg}$ twice a day (BID). Doses of $20 \mathrm{mg} / \mathrm{kg}$ are necessary to prevent the development of drug resistance, demonstrating dose dependence. The maximum tolerated dose (MTD) was defined as $450 \mathrm{mg}$ once daily, but the recommended Phase II dose was $300 \mathrm{mg}$ due to frequent dose-limiting toxicities. In BRAF inhibitor-naïve patients, the ORR was $60 \%$ and median PFS was 12.4 months ( $\mathrm{n}=18 ; 95 \%$ CI: 7.4 - not reached). In BRAF inhibitor pretreated patients, the ORR and median PFS were $22 \%$ and 1.9 months (95\% CI: 0.9-3.7) ${ }^{15}$ In Phase I trials, all 54 patients experienced at least one adverse effect. Thirty-eight of these patients (70.4\%) experienced serious grade $\geq 3$ adverse effects. The most common adverse effects were palmar-plantar erythrodysesthesia (PPED), hyperkeratosis, arthralgia, nausea, and pruritis. Of all, 9.3\% (5/54) of the patients experienced adverse effects that led to discontinuation of treatment (PPED, headache, hyperkeratosis, and neuralgia). Transient Bell's palsy was reported in $8 \%$ of the patients, but it has rarely been reported in association with other BRAF inhibitors..$^{15}$ Only one instance of squamous cell carcinoma $(3 \%-4 \%$ of the patients) was observed in the dose-expansion phase, which is lower than the observed rates with vemurafenib or dabrafenib therapy. Keratoacanthomas were not observed. ${ }^{15,19,20}$

As discussed earlier, monotherapy with BRAF inhibitors is known to induce neoplasia, most commonly $\mathrm{cSCC}^{21}$ at rates of $22 \%, 6 \%, 2.7 \%$ with vemurafenib, dabrafenib, and encorafenib treatments, respectively, due to paradoxical reactivation of the MAPK pathway with BRAF inhibition. ${ }^{22}$ The difference in the cSCC rates highlights an important characteristic of encorafenib illustrated here by the "paradox index". The "paradox index", introduced by Adelmann et al, ${ }^{22}$ is defined as the drug concentration that leads to $80 \%$ pERK activation (EC80) divided by the drug concentration that leads to $80 \%$ mutated BRAF inhibition (IC80) in BRAF-mutant melanoma cell lines. A higher index indicates superior inhibition of BRAF V600 with lesser pERK activity. Encorafenib, dabrafenib, and vemurafenib had paradox indices of 50, 10, and 5.5, respectively. ${ }^{22}$ Encorafenib's significantly greater paradox index is attributed to its extremely long dissociation time, which contributes to lower incidence of cSCC.

\section{Development of MEK inhibitors}

Understanding of the MAPK pathway in melanoma oncogenesis also led to the identification of MEK as a therapeutic target. BRAF mutations have been discussed extensively thus far, but another gain-of-function mutation identified in melanomas is NRAS. These two mutations are mutually exclusive but exert a similar downstream effect due to a common signaling pathway via the MEK-ERK cascade. Directly targeting RAS has been unsuccessful; however, downstream MEK inhibition has been shown to affect both BRAF-mutant and NRAS-mutant melanomas because MEK is the only kinase in the pathway that acts on ERK..$^{5,6,23}$ The response to MEK inhibition monotherapy has not been as robust as to BRAF inhibition; however, some clinical effects have been observed in clinical trials with trametinib and binimetinib. Trametinib (Mekinist ${ }^{\circledR}$, GSK112-212; GlaxoSmithKline plc), an orally available selective inhibitor of MEK1 and 2, was one of the first developed MEK inhibitors. A Phase III randomized trial compared trametinib with chemotherapy in patients with BRAFV600E/K-mutant melanoma with an overall response of $22 \%$ in the trametinib group and $8 \%$ in the chemotherapy group $(P=0.01)$. Median PFS was 4.8 months with trametinib monotherapy and 1.5 months with chemotherapy (HR $0.45,95 \%$ CI: $0.33-0.63, P<0.001$ ). At 6 months, the rate of overall survival (OS) in the trametinib group was $81 \%$, compared to $67 \%$ in the chemotherapy group $(P=0.01) .23,24$

\section{Binimetinib}

Binimetinib (Mektovi ${ }^{\circledR}$, MEK162; Array BioPharma) was a subsequently developed MEK inhibitor; it is a potent, selective, non-ATP-competitive allosteric inhibitor of MEK1 and 2. It is taken orally with rapid absorption; median time of maximal absorption is 1.48 hours. ${ }^{25}$ Food intake affects both concentration and time of absorption of binimetinib, as evaluated in the clinical study CMEK162A2103. ${ }^{25} \mathrm{~A}$ low-fat meal increased maximum plasma concentration $\left(\mathrm{C}_{\max }\right)$ by an average of $29 \%$, whereas a high-fat meal decreased $\mathrm{C}_{\max }$ by 
an average of $17 \%$, both compared to the fasting state. Both low- and high-fat food intake increased time to maximal absorption, however, did not affect the amount of binimetinib absorbed. Binimetinib is metabolized by several primary and secondary biotransformations; cytochrome 450 enzymes (CYP1A2 and CYP2C19) account for $17.8 \%$ of clearance via $\mathrm{N}$-demethylation, whereas direct glucuronidation accounts for approximately $62.2 \%$ of clearance. $62.3 \%$ of binimetinib is excreted in feces, $31.4 \%$ in urine, and $6.5 \%$ was excreted unchanged in urine. ${ }^{25}$

A Phase I trial with binimetinib was conducted by Bendell et al in 2011 and expanded to include data on biliary and colorectal cancer patients. Doses from $30 \mathrm{mg}$ to $80 \mathrm{mg}$ BID were administered to patients with different cancer types, including colorectal, pancreatic, and cholangiocarcinoma. Doselimiting toxicities occurred at doses of $80 \mathrm{mg}$ BID, including acneiform rash and central serous-like retinopathy, ${ }^{25,26}$ which resolved with lower dosages. Frequent AEs included gastrointestinal (GI) symptoms (diarrhea and nausea), skin rashes, peripheral edema, elevated creatine kinase, and retinal disorders. ${ }^{25}$ The expansion cohort was performed with a highest tolerated dose of $60 \mathrm{mg}$ BID in patients with biliary cancer, but the dose was subsequently decreased for higher than expected rates of ocular toxicity, predominantly central serous-like retinopathy. Ultimately, the expansion cohort was completed with $45 \mathrm{mg}$ BID. Overall, three of 91 evaluable patients in the Phase I study showed objective response to binimetinib therapy. ${ }^{25}$

Phase II trials of binimetinib were designed with three treatment arms: patients with BRAF- and NRAS-mutated tumors to receive $45 \mathrm{mg}$ BID and BRAF-mutated melanoma patients to receive $60 \mathrm{mg}$ BID. ${ }^{23}$ The majority of patients who received $45 \mathrm{mg}$ BID had been pretreated with some therapy prior to the trial. Partial response was confirmed in three patients with NRAS-mutated melanoma (10\%) and in two patients with BRAF-mutated melanoma (5\%). ORR was $20 \%$, with a median PFS of 4 months. ${ }^{23}$ AEs were similar to those described in Phase I trials. Grade 3 or 4 AEs occurred in four of 71 patients $(5.6 \%)$. Fifteen patients $(21 \%)$ discontinued treatment due to AEs and 33 patients (46\%) had at least one dose reduction after the onset of adverse reaction. Phase II trials were the first to confirm that binimetinib has activity, albeit a modest effect, in NRAS-mutated melanoma. ${ }^{23,27}$

The NEMO trial $^{6}$ is a Phase III study that studies the clinical effect of binimetinib on NRAS-mutant melanoma. Dummer et $\mathrm{al}^{6}$ compared binimetinib with chemotherapy in patients with unresectable or metastatic melanoma with an
NRAS mutation. Binimetinib showed superior median PFS of 2.8 months (95\% CI: 2.8-3.6) compared to 1.5 months (95\% CI: $1.5-1.7$ ) with dacarbazine (HR 0.62, 95\% CI: 0.47-0.80, $P<0.001)$. ORR was higher in the binimetinib arm, $15.2 \%$ (95\% CI: $11.2 \%-20.1 \%$ ) vs 6.8\% (95\% CI: 3.1\%-12.5\%); however, an interim analysis did not show significantly improved OS. 6,27 Therefore, it is unlikely for single-agent MEK inhibitors to move any further in the treatment of NRAS-mutant melanoma.

These studies, although limited, have paved the way for new studies investigating the utility of MEK inhibition. The now established role for MEK inhibition is part of combination therapy with BRAF inhibitors. The combination of BRAF and MEK inhibitors provides a more potent and durable inhibition of the MAPK pathway, and this is considered the standard of care targeted therapy as per the National Comprehensive Cancer Network (NCCN) and European Society for Medical Oncology (ESMO) guidelines in unresectable, metastatic BRAFV600-mutant melanoma. ${ }^{28,29}$ Administering these medications in combination slows the development of resistance as the tumor cannot proliferate via alternate pathways. ${ }^{11}$

\section{Combination therapy}

The first BRAF inhibitor and MEK inhibitor combination was dabrafenib and trametinib (D+T), which received FDA approval in 2014. ${ }^{3}$ Two Phase III studies comparing $\mathrm{D}+\mathrm{T}$ to dabrafenib ${ }^{30}$ and vemurafenib ${ }^{24}$ revealed a PFS of $11-11.4$ months and an ORR of $64 \%-69 \%$. The combination of vemurafenib and cobimetinib $(\mathrm{V}+\mathrm{C})$ showed comparable results: PFS 12.3 months and ORR 69.6\% (Table 1). ${ }^{31}$ No direct comparisons of combination therapy have been completed at this time. However, Daud et al performed an indirect treatment comparison of $\mathrm{D}+\mathrm{C}$ to $\mathrm{V}+\mathrm{C}$ using vemurafenib as the common comparator. The coBRIM (an international, multicenter, randomized Phase III trial of $\mathrm{V}+\mathrm{C}$ vs $\mathrm{V}+$ placebo in advanced melanoma patients) ${ }^{32}$ and COMBI-v (an international, multicenter, open-label, randomized Phase III trial of D+T vs $\mathrm{V}$ monotherapy in advanced melanoma patients) ${ }^{24}$ trials both used vemurafenib in the control arm, allowing for indirect comparison of the two combination therapies. They found no significant difference between efficacy of the two combinations. ${ }^{33}$ They also analyzed the safety profile for the two combinations and observed that D+T may have less toxicity than $\mathrm{V}+\mathrm{C}$. The incidence of treatment-related AEs (RR 0.92), incidence of any AE grade $\geq 3$ (RR 0.71), and incidence of dose interruption or modification (RR 0.77) were significantly lower with $\mathrm{D}+\mathrm{T}$ than $\mathrm{V}+\mathrm{C}$. The overall 
incidence of AEs (RR 0.98), any serious AEs (RR 0.84), or any AEs leading to death or discontinuation of therapy due to AE (RR 0.62) was not clinically significant between either combination. ${ }^{33}$ Overall, $\mathrm{V}+\mathrm{C}$ had higher incidence of minor AEs, whereas only chills, constipation, cough, and pyrexia occurred more frequently with $\mathrm{D}+\mathrm{T}$. Individual grade $\geq 3$ AEs were similar between both combinations. ${ }^{33}$

\section{Encorafenib and binimetinib combination therapy}

The combination of encorafenib and binimetinib was approved by the FDA on June 27, 2018, for the treatment of patients with unresectable or metastatic melanoma with BRAF V600E or V600K mutation. The approval was based on the COLUMBUS trial, a two-part, randomized, open-label Phase III trial that compared encorafenib plus binimetinib to encorafenib and vemurafenib monotherapy (Table 1). Key inclusion criteria included age 18 years or older; locally advanced (AJCC stage IIIb, IIIC, or IV), unresectable, or metastatic melanoma with a BRAF V600E/K mutation; ECOG performance status $<2$; and treatment naïve or have progressed after prior immunotherapy. Patients with untreated CNS lesions or uveal or mucosal melanoma were excluded. ${ }^{34}$

In the first part of the study, 577 patients were randomized equally into three groups to receive encorafenib $450 \mathrm{mg}$ once a day (QD) and binimetinib $45 \mathrm{mg}$ BID (COMBO450), encorafenib $300 \mathrm{mg}$ (ENCO300) QD, or vemurafenib $960 \mathrm{mg}$ BID (VEM). ENCO300 is the MTD from Phase I trials; ${ }^{15}$ however, the addition of binimetinib allowed for higher dose of $450 \mathrm{mg}$ without significant AEs as described subsequently. ${ }^{34}$ The primary end point was median PFS, which was significantly higher in the COMBO450 and ENCO groups compared to the monotherapy group; median PFS was 14.9 months in the COMBO450 group vs 9.6 months in the ENCO group, compared to 7.3 months for VEM by blinded central review. ${ }^{34}$ PFS for vemurafenib monotherapy was comparable with previous studies, as discussed previously, ${ }^{31,35}$ demonstrating markedly improved PFS data for encorafenib plus binimetinib. ORR confirmed by central review was $63 \%, 51 \%$, and $40 \%$ for COMBO450, ENCO, and VEM, respectively, whereas duration of response was 18.6 months, 15.2 months, and 12.3 months, respectively. ${ }^{34,36,37}$ Recently presented data at 18 months of follow-up also confirmed improved OS with COMBO450 therapy compared to VEM (33.6 months vs 16.9 months). ${ }^{37}$ Dose exposure information for COMBO 450 showed that $>80 \%$ of the patients were able to tolerate $\geq 80 \%$ relative dose intensity (RDI) of both encorafenib and binimetinib components of combination therapy. Median duration of exposure was 54.4 weeks to encorafenib $450 \mathrm{mg}$ QD and 53.8 weeks to binimetinib $45 \mathrm{mg}$ BID. In comparison, approximately $50 \%$ of the patients were able to tolerate $\geq 80 \%$ RDI of both ENCO300 and VEM over median treatment duration of 42.4 weeks for ENCO300 and 35.9 weeks for VEM. ${ }^{36}$

Higher dose exposure is likely attributed to better tolerated toxicity profile, allowing for the increased dose of treatment. In the COMBO450 cohort, grade $\geq 3$ AEs occurred in $58 \%$ of the patients, compared to $66 \%$ and $63 \%$ in the ENCO300 and VEM groups. The median time to the first grade $\geq 3 \mathrm{AE}$ was 2.5 months for COMBO450, 0.4 months for ENCO300, and 1.3 months for VEM. Typical BRAF inhibitor-related AEs, such as arthralgias and hyperkeratosis, were observed less frequently with the addition of an MEK inhibitor. Major AEs were primarily typical MEK inhibitorassociated AEs, such as elevated creatine kinase and ocular toxicity. These occurred less frequently than in binimetinib monotherapy. Eight percentage of the COMBO450 patients discontinued treatment due to AEs, compared to $12 \%$ and $14 \%$ in the ENCO300 and VEM groups. Patients in the COMBO450 group tolerated severe AEs for a median of 3.8 months, compared to 1.8 months for both ENCO300 and VEM. ${ }^{36}$

Evaluation of data from part 1 also demonstrated that ENCO300 alone improved PFS compared to VEM. The second part of the COLUMBUS trial ${ }^{38}$ evaluated the contribution of binimetinib to combination therapy by comparing the same ENCO300 monotherapy arm to encorafenib $300 \mathrm{mg}$ QD plus binimetinib $45 \mathrm{mg}$ BID (COMBO300). Three hundred forty-four patients were randomized into the two groups, the ENCO300 group composed of a subset of patients continuing ENCO300 from part 1 of the study. Analysis of data by blinded central review again showed superior PFS with combination therapy, as PFS for COMBO300 was 12.9 months compared to PFS of the entire ENCO300 cohort (both parts 1 and 2 of study) of 9.2 months (with PFS of 7.4 months of only the ENCO300 patients added in part 2). By central review, ORRs were $66 \%, 50 \%$, and $50 \%$ for $\mathrm{COMBO} 300$, ENCO300 (parts 1+2), and ENCO300 (part 2). Median duration of response was 12.7 months, 12.9 months, and 7.5 months, respectively. ${ }^{38}$ These results clearly demonstrate the contribution of binimetinib to encorafenib therapy. The differences in PFS and duration of response between the entire ENCO300 cohort as compared to the subset analyzed in part 2 alone are likely due to a shorter duration of therapy in the latter subset. 
As in part 1, combination therapy achieved a dramatically higher RDI. In the COMBO300 cohort, $88.7 \%$ of the patients tolerated $80 \%-100 \%$ RDI of encorafenib $300 \mathrm{mg}$ and $82.9 \%$ RDI of binimetinib $45 \mathrm{mg}$ BID over 47.6 weeks of combination therapy. Of those patients, $53.7 \%$ and $47.1 \%$ tolerated $100 \%$ RDI of encorafenib and binimetinib, respectively. ENCO300 monotherapy only achieved RDI of $80 \%-100 \%$ in $52.2 \%$ of the patients over a median of 43.8 weeks. ${ }^{38}$ Serious AEs occurred in $29 \%, 33 \%$, and $30 \%$, respectively, in COMBO300, ENCO300 parts 1+2, and ENCO300 part 2. Twelve percentage, $13 \%$, and $10 \%$ of the patients experienced AEs that lead to treatment discontinuation, whereas $45 \%, 69 \%$, and $63 \%$ of the patients, respectively, had dose interruptions or changes. GI symptoms (nausea, diarrhea, and vomiting), arthralgia, fatigue, and elevated CK were among the most common $\mathrm{AEs}$ in the $\mathrm{COMBO} 300$ group, whereas the ENCO300 group had more prevalent skin-related conditions: arthralgia and myalgia. ${ }^{38}$ Overall, the median duration of treatment with COMBO450 was 51.2 weeks and 52.1 weeks with COMBO300, and both groups had $98 \%$ incidence of AEs. COMBO450 had more severe AEs than COMBO300 at $58 \%$ compared to $47 \%$ of the patients. However, this did not lead to significant increase in discontinuation of treatment. In both groups, $13 \%$ of the patients discontinued treatment due to AEs, whereas $48 \%$ and $45 \%$ had dose interruptions or changes (COMBO450 and COMBO300, respectively). ${ }^{38}$ No new safety concerns were found at 18 -month follow-up. ${ }^{37}$

Clearly, binimetinib has meaningful contribution to combination therapy as there is improvement in both PFS and ORR in COMBO300 vs ENCO300. Combination therapy in both parts results in higher RDI, fewer serious AEs, and fewer AEs leading to discontinued therapy. When comparing COMBO450 and $\mathrm{COMBO} 300, \mathrm{COMBO} 450$ demonstrated longer PFS and duration of response. Coupled with a comparable toxicity profile, the higher dose of encorafenib in COMBO450 does not seem to increase the incidence of AEs. The higher dose of encorafenib may contribute to improved efficacy of encorafenib plus binimetinib combination therapy. . $^{34,36,38}$

\section{Future directions}

At present, there is no consensus on the sequencing of available metastatic melanoma treatments with regard to BRAF-targeted therapy and immunotherapies. There are ongoing clinical trials investing the sequencing of BRAF/ MEK inhibitors, how they fit in with immunotherapy, and how effective combination treatments may be ${ }^{39,40}$ At present, the NCCN and ESMO guidelines support the use of anti-PD1 immunotherapy for all types of metastatic melanomas and
BRAF/MEK inhibitors for BRAFV600-mutated disease. ${ }^{28,29}$ Furthermore, the use of BRAF-targeted therapy has also moved to the neoadjuvant and stage 3 adjuvant setting in melanoma, and encorafenib and binimetinib will likely be explored in these settings as well. While the current research is encouraging and has benefited the lives of many patients, the rate of acquired resistance remains a concern with BRAF-targeted therapy. However, similar to immunotherapy, durable responses and long-lasting benefits are certainly possible with targeted therapy, supported by a recently published 5-year follow-up ${ }^{41}$ of a Phase II trial ${ }^{42}$ with the findings of a 5 -year OS of $28 \%$ and a PFS of $14 \%$ in patients treated with $\mathrm{D}+\mathrm{T}$.

\section{Conclusion}

Given its high response rate, impressive PFS, and favorable toxicity profile, encorafenib and binimetinib will likely become a very frequently used BRAF/MEK inhibitor therapy. The pharmacokinetics of encorafenib renders it unique from the other BRAF inhibitors with a notable PFS and ORR and delayed onset of resistance, although of course there are no trials that directly compare encorafenib and binimetinib to the other two BRAF/MEK inhibitor combinations. Currently ongoing clinical trials and studies are evaluating the wealth of knowledge gained from understanding BRAF and MEK inhibition and their role in the treatment of melanoma. A new third generation of "paradox breaker" BRAF inhibitors ${ }^{43}$ such as PLX7904 (preclinical; BioVision Inc, San Francisco, CA, USA) and PLX8394 ${ }^{44}$ (NCT02428712; Plexxikon) that escape the cycle of acquired resistance are under investigation. The role of encorafenib and binimetinib in combination with other new classes of medications is currently under investigation. There is particular interest in combination with immunotherapeutics, such as the SECOMBIT trial $^{45}$ (NCT02631447), investigating the appropriate therapeutic sequencing of the anti-CTLA4 antibody ipilimumab (Yervoy ${ }^{\circledR}$, L01XC11; Bristol-Myers Squibb, New York City, NY, USA) and encorafenib/binimetinib. Another study, the IMMU-TARGET trial ${ }^{46}$ (NCT02902042), is investigating the combination of encorafenib/binimetinib with another immunotherapeutic, the anti-PD1 antibody pembrolizumab (Keytruda $^{\circledR}$, L01XC18; Merck \& Co., Inc., Whitehouse Station, NJ, USA). Finally, the LOGIC- 2 trial ${ }^{47}$ (NCT02159066) has been ongoing, which is investigating the combination of encorafenib and binimetinib with other classes of medications not yet applied to melanoma treatment, such as CDK4/6, FGFR, c-Met, and PI3K inhibitors. While the results of these studies may allow for further elucidation of optimal 
treatments for patients with melanoma, it is undeniable that the FDA approval of nine new drugs for metastatic melanoma since 2011 has significantly changed the landscape of available treatments for these patients who previously had minimal treatment options.

\section{Disclosure}

JS has no conflicts of interest to disclose related to this work. $\mathrm{ZE}$ is a member of the Medical Advisory Board for Array BioPharma and Regeneron, and JSZ is a member of the Medical Advisory Board for Delcath Systems. He also receives grant funding from Delcath Systems. He is a primary investigator on clinical trials funded by Delcath Systems, Amgen, Castle Biosciences, Provectus, Philogen, and Novartis. He consults for Amgen, Castle Biosciences, and Philogen. He is a member of the speaker's bureau for Amgen, Array BioPharma, and Sun Pharma. Finally, he has participated on advisory boards for Amgen, Merck \& Co., Inc., and Array BioPharma. The authors report no other conflicts of interest in this work.

\section{References}

1. Davies H, Bignell GR, Cox C, et al. Mutations of the BRAF gene in human cancer. Nature. 2002;417(6892):949-954.

2. Ascierto PA, Kirkwood JM, Grob JJ, et al. The role of BRAF V600 mutation in melanoma. $J$ Transl Med. 2012;10(1):85-89.

3. Karoulia Z, Gavathiotis E, Poulikakos PI. New perspectives for targeting RAF kinase in human cancer. Nat Rev Cancer. 2017;17(11): 676-691.

4. Eroglu Z, Ribas A. Combination therapy with BRAF and MEK inhibitors for melanoma: latest evidence and place in therapy. Ther Adv Med Oncol. 2016;8(1):48-56.

5. Solit DB, Garraway LA, Pratilas CA, et al. BRAF mutation predicts sensitivity to MEK inhibition. Nature. 2006;439(7074):358-362.

6. Dummer R, Schadendorf D, Ascierto PA, et al. Binimetinib versus dacarbazine in patients with advanced NRAS-mutant melanoma (NEMO): a multicentre, open-label, randomised, phase 3 trial. Lancet Oncol. 2017;18(4):435-445.

7. Chappell WH, Steelman LS, Long JM, et al. Ras/Raf/MEK/ERK and $\mathrm{PI} 3 \mathrm{~K} / \mathrm{PTEN} / \mathrm{Akt} / \mathrm{mTOR}$ inhibitors: rationale and importance to inhibiting these pathways in human health. Oncotarget. 2011;2(3):135-164.

8. Tsai J, Lee JT, Wang W, et al. Discovery of a selective inhibitor of oncogenic B-Raf kinase with potent antimelanoma activity. Proc Natl Acad Sci U S A. 2008;105(8):3041-3046.

9. Bollag G, Hirth P, Tsai J, et al. Clinical efficacy of a RAF inhibitor needs broad target blockade in BRAF-mutant melanoma. Nature. 2010; 467(7315):596-599.

10. Chapman PB, Hauschild A, Robert C, et al. Improved survival with vemurafenib in melanoma with BRAF V600E mutation. $N$ Engl J Med. 2011;364(26):2507-2516.

11. Griffin M, Scotto D, Josephs DH, et al. BRAF inhibitors: resistance and the promise of combination treatments for melanoma. Oncotarget. 2017;8(44):78174-78192.

12. Hauschild A, Grob JJ, Demidov LV, et al. Dabrafenib in BRAF-mutated metastatic melanoma: a multicentre, open-label, phase 3 randomised controlled trial. Lancet. 2012;380(9839):358-365.

13. Su F, Viros A, Milagre C, et al. RAS mutations in cutaneous squamouscell carcinomas in patients treated with BRAF inhibitors. $N$ Engl J Med. 2012;366(3):207-215.
14. Sullivan RJ, Flaherty KT. Resistance to BRAF-targeted therapy in melanoma. Eur J Cancer. 2013;49(6):1297-1304.

15. Delord JP, Robert C, Nyakas M, et al. Phase I dose-escalation and -expansion study of the BRAF inhibitor encorafenib (LGX818) in metastatic BRAF-mutant melanoma. Clin Cancer Res. 2017;23(18): $5339-5348$

16. Holderfield M, Merritt H, Chan J, et al. RAF inhibitors activate the MAPK pathway by relieving inhibitory autophosphorylation. Cancer Cell. 2013;23(5):594-602.

17. Stuart DD, Li N, Poon DJ, et al. Abstract 3790: preclinical profile of LGX818: A potent and selective RAF kinase inhibitor. Cancer Res. 2012;72(8 Supplement):3790

18. Koelblinger P, Thuerigen O, Dummer R. Development of encorafenib for BRAF-mutated advanced melanoma. Curr Opin Oncol. 2018;30(2): $1-133$.

19. Ascierto PA, Minor D, Ribas A, et al. Phase II trial (BREAK-2) of the BRAF inhibitor dabrafenib (GSK2118436) in patients with metastatic melanoma. J Clin Oncol. 2013;31(26):3205-3211.

20. Mcarthur GA, Chapman PB, Robert C, et al. Safety and efficacy of vemurafenib in $\mathrm{BRAF}(\mathrm{V} 600 \mathrm{E})$ and $\mathrm{BRAF}(\mathrm{V} 600 \mathrm{~K})$ mutation-positive melanoma (BRIM-3): extended follow-up of a phase 3, randomised, open-label study. Lancet Oncol. 2014;15(3):323-332.

21. Dummer R, Robert C, Nyakas M. Initial results from a phase I, openlabel, dose escalation study of the oral BRAF inhibitor LGX818 in patients with BRAF V600 mutant advanced or metastatic melanoma. J Clin Oncol. 2013;31Suppl:Abstr 9028.

22. Adelmann $\mathrm{CH}$, Ching $\mathrm{G}$, du L, et al. Comparative profiles of BRAF inhibitors: the paradox index as a predictor of clinical toxicity. Oncotarget. 2016;7(21):30453-30460.

23. Ascierto PA, Schadendorf D, Berking C, et al. MEK162 for patients with advanced melanoma harbouring NRAS or Val600 BRAF mutations: a non-randomised, open-label phase 2 study. Lancet Oncol. 2013; 14(3):249-256.

24. Robert C, Karaszewska B, Schachter J, et al. Improved overall survival in melanoma with combined dabrafenib and trametinib. N Engl J Med. 2015;372(1):30-39.

25. Koelblinger P, Dornbierer J, Dummer R. A review of binimetinib for the treatment of mutant cutaneous melanoma. Future Oncol. 2017;13(20): $1755-1766$.

26. Bendell JC, Javle M, Bekaii-Saab TS, et al. A phase 1 dose-escalation and expansion study of binimetinib (MEK162), a potent and selective oral MEK1/2 inhibitor. Br J Cancer. 2017;116(5):575-583.

27. Luke JJ, Flaherty KT, Ribas A, Long GV. Targeted agents and immunotherapies: optimizing outcomes in melanoma. Nat Rev Clin Oncol. 2017;14(8):463-482.

28. NCCN: National Comprehensive Cancer Network. Clinical Practice Guidelines in Oncology, Melanoma Version 2. Fort Washington, PA: National Comprehensive Cancer Network; 2018.

29. Dummer R, Hauschild A, Lindenblatt N, Pentheroudakis G, Keilholz U; ESMO Guidelines Committee. Cutaneous melanoma: ESMO Clinical Practice Guidelines for diagnosis, treatment and follow-up. Ann Oncol. 2015;26(suppl_5):v126-v132.

30. Long GV, Flaherty KT, Stroyakovskiy D, et al. Dabrafenib plus trametinib versus dabrafenib monotherapy in patients with metastatic BRAF $\mathrm{V} 600 \mathrm{E} / \mathrm{K}$-mutant melanoma: long-term survival and safety analysis of a phase 3 study. Ann Oncol. 2017;28(7):1631-1639.

31. Ascierto PA, Mcarthur GA, Dréno B, et al. Cobimetinib combined with vemurafenib in advanced BRAF(V600)-mutant melanoma (coBRIM): updated efficacy results from a randomised, double-blind, phase 3 trial. Lancet Oncol. 2016;17(9):1248-1260.

32. Larkin J, Ascierto PA, Dréno B, et al. Combined vemurafenib and cobimetinib in BRAF-mutated melanoma. $N$ Engl J Med. 2014;371(20): $1867-1876$

33. Daud A, Gill J, Kamra S, Chen L, Ahuja A. Indirect treatment comparison of dabrafenib plus trametinib versus vemurafenib plus cobimetinib in previously untreated metastatic melanoma patients. $J$ Hematol Oncol. 2017;10(1):23. 
34. Dummer R, Ascierto PA, Gogas HJ, et al. Encorafenib plus binimetinib versus vemurafenib or encorafenib in patients with BRAF-mutant melanoma (COLUMBUS): a multicentre, open-label, randomised phase 3 trial. Lancet Oncol. 2018;19(5):603-615.

35. Long GV, Stroyakovskiy D, Gogas H, et al. Dabrafenib and trametinib versus dabrafenib and placebo for Val600 BRAF-mutant melanoma: a multicentre, double-blind, phase 3 randomised controlled trial. Lancet. 2015;386(9992):444-451.

36. Dummer R, Ascierto PA, Gogas HJ. Results of COLUMBUS part 1: a phase 3 trial of encorafenib (ENCO) plus binimetinib (BINI) versus vemurafenib (VEM) or ENCO in BRAF-mutant melanoma. In: SMR Annual Meeting. November 6-9, 2016, Boston, MA.

37. Dummer R, Ascierto PA, Gogas HJ. Overall Survival in COLUMBUS: A Phase 3 Trial of Encorafenib (ENCO) Plus Binimetinib (BINI) vs Vemurafenib (VEM) or ENCO in BRAF-Mutant Melanoma. J Clin Oncol. 2018;(Suppl): abstr 9504.

38. Dummer R, Ascierto PA, Gogas H, et al. Results of COLUMBUS Part 2: A phase 3 trial of encorafenib (ENCO) plus binimetinib (BINI) versus ENCO in BRAF-mutant melanoma. Annals of Oncology. 2017;28(suppl_5).

39. Ribas A, Hodi FS, Lawrence DP, et al. Pembrolizumab (pembro) in combination with dabrafenib (D) and trametinib (T) for $B R A F$-mutant advanced melanoma: Phase 1 KEYNOTE-022 study. Journal of Clinical Oncology. 2016;34(15_suppl):3014.

40. Sullivan RJ, Gonzalez R, Lewis KD, et al. Atezolizumab (A) + cobimetinib (C) + vemurafenib (V) in BRAFV600-mutant metastatic melanoma (mel): Updated safety and clinical activity. Journal of Clinical Oncology. 2017;35(15_suppl):3063.
41. Long GV, Eroglu Z, Infante J, et al. Long-term outcomes in patients with BRAF V600-mutant metastatic melanoma who received dabrafenib combined with trametinib. J Clin Oncol. 2018;36(7):667-673.

42. Long GV, Weber JS, Infante JR, et al. Overall Survival and Durable Responses in Patients With BRAF V600-Mutant Metastatic Melanoma Receiving Dabrafenib Combined With Trametinib. J Clin Oncol. 2016; 34(8):871-878.

43. Zhang C, Spevak W, Zhang Y, et al. RAF inhibitors that evade paradoxical MAPK pathway activation. Nature. 2015;526(7574) 583-586.

44. ClinicalTrials.gov [homepage on the Internet]. A Study of PLX8394 as a Single Agent in Patients With Advanced Unresectable Solid Tumors. Available from: https://clinicaltrials.gov/ct2/show/NCT02428712. Accessed October 16, 2018.

45. ClinicalTrials.gov [homepage on the Internet]. Sequential Combo Immuno and Target Therapy (SECOMBIT) Study (SECOMBIT). Available from: https://clinicaltrials.gov/ct2/show/NCT02631447. Accessed October 16, 2018.

46. ClinicalTrials.gov [homepage on the Internet]. Open Label, Multicentre Study of Encorafenib + Binimetinib + PD (Programmed Cell Death Protein)-1 Antibody Pembrolizumab (IMMU-TARGET). Available from: https://clinicaltrials.gov/ct2/show/NCT02902042. Accessed October 16, 2018.

47. ClinicalTrials.gov [homepage on the Internet]. Array BioPharma. LGX818 and MEK162 in Combination with a third Agent (BKM120, LEE011, BGJ398 or INC280) in Advanced BRAF Melanoma (LOGIC-2). Available from: https://clinicaltrials.gov/ct2/show/ NCT02159066. Accessed October 16, 2018.
OncoTargets and Therapy

\section{Publish your work in this journal}

OncoTargets and Therapy is an international, peer-reviewed, open access journal focusing on the pathological basis of all cancers, potential targets for therapy and treatment protocols employed to improve the management of cancer patients. The journal also focuses on the impact of management programs and new therapeutic agents and protocols on

\section{Dovepress}

patient perspectives such as quality of life, adherence and satisfaction. The manuscript management system is completely online and includes a very quick and fair peer-review system, which is all easy to use. Visit http://www.dovepress.com/testimonials.php to read real quotes from published authors. 